\title{
COVID-19 Vaccination in the Wake of a Fourth Wave of the Pandemic: an Evidence- Based Strategy is Desperately Needed
}

Murat Akova

Editor-in-Chief, Infectious Diseases and Clinical Microbiology

Department of Infectious Diseases and Clinical Microbiology, Hacettepe University School of Medicine, Ankara, Turkey

A s of August 21, 2021, more than 32\% of the world population has received at least one dose of the COVID-19 vaccine and almost one fourth is fully vaccinated. However, only $1.4 \%$ of the population in the developing world have received at least one dose (1). While the pandemic ravages the unvaccinated communities, a new wave of the disease is emerging in countries with high rates of full vaccination status (2, 3). There may be at least three factors responsible for the latter situation:

1. Emerging new variants (e.g. delta) which are much higher contagious than their predecessors and more resistant to antibodies produced by both natural disease and the available vaccines

2. Relaxing the preventive measures (e.g. use of masks, public gatherings) in the community mostly due to initial confidence provided by vaccination

3. Waning immunity from the initial course of vaccination

Although the term describing the current situation as "the pandemic of the unvaccinated" is appropriate since most severe cases and a significant portion of mortality still occur in unvaccinated populations, the recent reports indicating that fully vaccinated people can also get infected with high viral loads are worrisome (4). The fact that different vaccines can produce varying types and strengths of immunity makes it more complicated to offer an evidence-based vaccination strategy.

In a letter published in this issue of the Journal, Azap, et al. (5) reported that among those 62 healthcare workers (HCWs) who caught COVID-19 in their institution, 39 (63\%) were fully vaccinated with CoronaVac (an inactivated SARS-CoV-2 vaccine by Sinovac, China). All had mild disease, and no mortality was reported. No SARS-CoV-2 variant information was available, but presumably, the alpha variant was dominant in Turkey during the reporting period, and the delta variant might be emerging. The only placebo-controlled, randomized study with CoronaVac reported $83.5 \%$ overall efficacy and $100 \%$ protection from mortality for a median follow-up of 43 days (6). The real-world data with CoronaVac from Chile indicated a $66 \%$ vaccine efficacy for symptomatic disease,
Corresponding Author: Murat Akova

E-mail:

makova@hacettepe.edu.tr

Published: August 31, 2021

Suggested citation: Akova M. COVID-19 Vaccination in the wake of a fourth wave of the pandemic: an evidencebased strategy is desperately needed. Infect Dis Clin Microbiol 2021; 2: 52-54.

DOI: $10.36519 / \mathrm{idcm} .2021 .82$ 
90\% for intensive care unit admission and $86 \%$ for prevention of mortality (7). Full immunization with CoronaVac was also highly effective for preventing severe disease and mortality when used in a local epidemic caused by the delta variant in China (8). When put together, these data indicate CoronaVac can provide strong prevention from severe disease and mortality, at least for the short term. Unpublished observations in Turkey lend support to these findings (9).

The delta variant has become dominant and the time elapsed from the initial vaccination among at least some of the vaccinees exceeds eight months; the whole scenario is changing now. The recent reports indicate that the delta variant can also infect those who are fully vaccinated, and the viral load is comparable between fully and non-vaccinated among mRNA-based vaccine recipients (2-4). Claiming the waning immunity after a while from the vaccination, several countries, including Israel, France and the US, have initiated a third dose campaign first within the immunocompromised population, now extending to the whole community. In Turkey, the situation is somewhat different. Turkey has started community vaccination in mid-January 2021 with CoronaVac and Pfizer- BioNTech vaccine became available in early April. Thus, more than six months elapsed after the full vaccination schedule of early vaccinees and anecdotal reports indicate breakthrough infections among this group, some severe in nature and with mortality. Therefore, Turkey's Ministry of Health offered a third dose of vaccine to all HCWs and those older than 50 years who were initially vaccinated with two-dose CoronaVac. The third dose was provided either as homologous (with CoronaVac) or heterologous (with Pfizer-BioNTech) vaccination. Those who acquired the disease more than three months ago or earlier are also eligible for a two-course vaccination schedule with a vaccine of their preference. Finally, a highly debatable and first-ever-in-its-kind suggestion has come from the Turkish Ministry of Health; the fourth dose of vaccine is suggested for those who had two-dose CoronaVac and a third dose of the Pfizer-BioNTech vaccine. Although there is no scientific basis for this offer (as acknowledged by the Minister of Health himself), it is a prerequisite for those who travel to countries where CoronaVac is not an approved COVID-19 vaccine, but the two-course schedule of the Pfizer-BioNTech vaccine is counted eligible for full vaccination. There is no evidence for the safety of such an application; however, there is data in those who initially acquired disease and were later injected with the Pfizer-BioNTech vaccine with a single or two jabs. The data indicated that while the first dose of the vaccine augmented neutralizing antibody levels significantly, the second dose failed to increase further (10). Thus, if the initial two doses of CoronaVac were considered to imitate the immune priming by the natural infection, a four-dose schedule may prove useless, particularly when given with short intervals.

Since no head-to-head comparisons of different vaccines have been available so far, it is scientifically unjustified to compare the efficacy of different COVID-19 vaccines. However, one can assume that since the level of initial production of neutralizing antibodies by inactivated vaccines is much lower than those with mRNA vaccines, the decline of neutralizing activity by time with inactivated vaccines may occur earlier (10). The real-life observations of vaccine efficacy may clarify this issue and provide evidence of the timing of the booster dose(s). In that respect, Turkey has a unique opportunity to provide evidence on CoronaVac efficacy and its changing course over time since a large population were fully vaccinated with this vaccine more than six months ago. The detailed analysis of vaccine efficacy and making the data publicly available will increase public confidence about vaccination schedules offered and undoubtedly will help decrease vaccine hesitancy in the community. 


\section{REFERENCES}

1 Ritchie H, Mathieu E, Rodés-Guirao L, Appel C, Giattino C, Ortiz-Ospina E, et al. Coronavirus (COVID-19) vaccinations [Internet]. Published online at OurWorldInData.org. (cited August 21, 2021) Available from: https://ourworldindata.org/ covid-vaccinations

2 Kupferschmidt K. Evolving Threat: New variants have changed the face of pandemic. What will the virus do next? [Internet]. Science. (August 19, 2021; cited August 21, 2021 Available from: https://www.sciencemag.org/news/2021/08/ new-sars-cov-2-variants-have-changed-pandemic-what-willvirus-do-next

3 Wadman M. A grim warning from Israel: Vaccination blunts, but not defeat delta [Internet]. Science. (August 16, 2021; cited August 21, 2021) Available from: https://www.sciencemag.org/news/2021/08/grim-warning-israel-vaccination-blunts-does-not-defeat-delta

4 Pouwels K, Pritchard E, Matthews PC, Stoesser N, Eyre DW, Vihta KD, et al. Impact of Delta on viral burden and vaccine effectiveness against new SARS-CoV-2 infections in the UK. [Internet] (August 16, 2021; cited August 21, 2021 Available from: https:/www.ndm.ox.ac.uk/files/coronavirus/ covid-19-infection-survey/finalfinalcombinedve20210816.pdf

5 Azap-Kurt Ö, Arslan H, Erol Ç, Sarı N. Healthcare workers should be vaccinated with the highest effective vaccine available. Infect Dis Clin Microbiol. 2021;2: 107-8. [CrossRef]

6 Tannover MD, Doğanay HL, Akova M, Güner HR, Azap A, Akhan S, et al. Efficacy and safety of an inactivated whole-vi- rion SARS-CoV-2 vaccine (CoronaVac): interim results of a double-blind, randomised, placebo-controlled, phase 3 trial in Turkey. The Lancet. 2021;398:213-22. [CrossRef]

7 Jara A, Undurraga EA, González C, Paredes F, Fontecilla T, Jara $\mathrm{G}$, et al. Effectiveness of an inactivated SARS-CoV-2 vaccine in Chile. N Engl J Med. Sep 2, 2021;385(10):875-88. [CrossRef]

8 Kang M, Yi Y, Li Y, Sun L, Deng A, Hu T, et al. Effectiveness of inactivated COVID-19 vaccines against COVID-19 pneumonia and severe illness caused by the B.1.617.2 (Delta) variant: Evidence from an outbreak in Guangdong, China [Internet]. SSRN. (August 5, 2021; cited August 21, 2021) Available from: https://papers.ssm.com/sol3/papers.cfm?abstract_ $\underline{i d=3895639}$

9 Aşılama çalışmalan etkisini gösteriyor [Internet]. TRT Haber. (April 13, 2021; cited August 21, 2021). Turkish. Available from: https://www.trthaber.com/haber/saglik/asilama-calismalari-etkisini-gosteriyor-572547.html

10 Anderson M, Stec M, Rewane A, Landay A, Cloherty G, Moy J. SARS-CoV-2 antibody responses in infection-naive or previously infected individuals after 1 and 2 doses of the BNT162b2 vaccine. JAMA Netw Open. 2021;4(8):e2119741 [CrossRef]

11 Khoury DS, Cromer D, Reynaldi A, Schlub TE, Wheatley AK, Juno JA, et al. Neutralizing antibody levels are highly predictive of immune protection from symptomatic SARS-CoV-2 infection. Nat Med 2021;27:1205-11 [CrossRef] 